\title{
ANÁLISE COMPARATIVA ENTRE TIPOS DE ALIMENTAÇÃO DE MICRURUS CORALLINUS (SERPENTES, ELAPIDAE) EM CATIVEIRO'
}

\author{
Eliana de Oliveira Serapicos ${ }^{2}$ \\ José Luiz Bernardino Merusse ${ }^{3}$
}

\begin{abstract}
COMPARATIVE ANALYSIS BETWEEN FEEDING TYPES MICRURUS CORALLINUS (SERPENTES, ELAPIDAE) IN CAPTIVITY. Micrurus corallinus (Merrem, 1820) kept in laboratory animal rooms swallow voluntarily various colubrid species. The most accepted species belong to the genera Sibynomorphus Fitzinger, 1843, Oxyrhopus Wagler, 1830 and Liophis Wagler, 1830. The voluntary feeding guaranteed resistance against pathogenic agents. The forced feeding was unsatisfactory due to stress resulting from handling, feeding frequency (30 days), susceptibility to pathogenic agents and high occurrence of pathologies.
\end{abstract}

KEYWORDS. Micrurus, feeding, handling, laboratory animals rooms.

\section{INTRODUÇÃO}

A dieta de Micrurus corallinus (Merrem, 1820) em ambiente natural é especializada, compreendendo anfisbenídeos, gimnofionos, lagartos e colubrídeos (GANs, 1969, 1974; Roze, 1996; MARques \& SAZIMA, 1997). Em cativeiro, é reportada a utilização de alimentos congelados (Softly \& CocketT, 1966) e recém-mortos (Marques \& SAZima, 1997). A rejeição a estes tipos de alimentos leva à indução dos mesmos, como um procedimento freqüentemente utilizado em serpentes mantidas em cativeiro. Vários aspectos negativos já foram relatados, como estresse e regurgitação (ASHLEY \& BURCHFIELD, 1968) e aumento da susceptibilidade à ação dos agentes patogênicos (Mishima et al., 1972).

O objetivo foi realizar um estudo comparativo entre dois tipos de alimentação, voluntária e forçada, a fim de aprimorar o manejo de M. corallinus em biotério.

\footnotetext{
1. Parte da Dissertação de Mestrado da autora sênior, Programa de Pós- Graduação em Patologia Experimental e Comparada, Departamento de Patologia, Faculdade de Medicina Veterinária e Zootecnia, Universidade de São Paulo.

2. Laboratório de Herpetologia, Instituto Butantan, Av. Vital Brazil, 1500, 05503-900, São Paulo, SP, Brasil. (serapicos@butantan.gov.br)

3. Departamento de Patologia, Faculdade de Medicina Veterinária e Zootecnia, Universidade de São Paulo, Av. Prof. Dr. Orlando Marques de

Paiva, 87, 05508-900, São Paulo, SP, Brasil. (merusse@usp.br)
} 


\section{MATERIAL E MÉTODOS}

Os animais foram inicialmente mantidos em quarentena por um período de 60 dias sob cuidados veterinários. As condições ambientais da quarentena eram semelhantes às da sala de manutenção, onde a temperatura média mensal variou entre $22,3 \pm 1,6$ e $28,8 \pm 1,4^{\circ} \mathrm{C}$ e os valores médios obtidos para a umidade relativa variaram entre $66,3 \pm 11,0$ e $91,1 \pm 4,4 \%$. Durante o período de quarentena todos os animais receberam alimentação forçada. As caixas de manutenção foram confeccionadas em madeira e tela, medindo $800 \mathrm{~cm}^{2}$. O interior foi forrado com papelão e utilizado substrato vegetal úmido e estéril, e um recipiente com água ad libitum.

Durante um período de 15 meses foram utilizados 32 exemplares de M. corallinus (fig. 1) divididos em 2 grupos experimentais, os quais receberam alimentação distinta a partir da entrada na sala de manutenção. Para o grupo da alimentação voluntária (A) foram oferecidas, mensalmente, várias espécies de colubrídeos (Apostolepis assimilis Reinhardt, 1861; Atractus reticulatus Boulenger, 1885; Helicops modestus Günther, 1861; Liophis milliaris Linnaeus, 1758; L. poecilogyrus Wied, 1825; L. typhlus Linnaeus, 1758; Oxyrhopus guibei Duméril \& Bibron Duméril, 1854; Sibynomorphus mikanii Schlegel, 1837 e S. neuwiedii Ihering, 1910) e uma espécie de anguídeo (Sauria), Ophiodes striatus Spix,

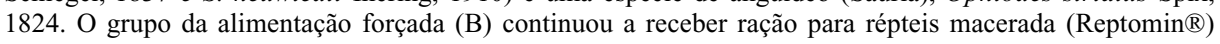
associada a soro glicosado a $5 \%$, mensalmente, através de sonda, por via esofágica, sendo anestesiados com $\mathrm{CO}_{2}$.

O peso dos espécimens oferecidos para o grupo A variou entre 5 e $20 \%$ do peso das $M$. corallinus. Ressalta-se que os exemplares com valores próximos de $5 \%$ muitas vezes eram rejeitados e os próximos de $20 \%$ não eram ingeridos por $M$. corallinus. Para o grupo $\mathrm{B}$, a quantidade de alimentação administrada também variou de acordo com o peso da serpente, sendo $5 \%$ o valor de referência para cálculo. Valores acima de 5\% provocaram regurgitação.

\section{RESULTADOS E DISCUSSÃO}

Micrurus corallinus aceitou a alimentação oferecida (colubrídeos) em biotério, embora seja diferente daquela de sua preferência em ambiente natural. Os exemplares de colubrídeos mais aceitos foram as espécies de Sibynomorphus Fitzinger, $1843(65,8 \%)$, Oxyrhopus Wagler, 1830 (60,0\%) e Liophis Wagler, 1830 (54,2\%), sendo que nenhum destes apresenta hábito fossorial. MARQUes \& SAZIMA (1997) identificaram Xenodon neuwiedii (Günther, 1863) na dieta de M. corallinus em ambiente natural e AMARAL (1978) identificou Atractus sp. para a mesma espécie, em cativeiro.

A relação entre a oferta e a aceitação da dieta oferecida em biotério foi considerada satisfatória, sendo superior a 50\% no total do grupo abrangendo machos e fêmeas. Em relação ao sexo, os machos aceitaram melhor a alimentação voluntária, sendo de $70 \%$ para as espécies de Sibynomorphus, 62,5\% para as de Oxyrhopus e 60\% para aquelas de Liophis e nas fêmeas foi de $50,0 \%, 42,9 \%$ e $44,4 \%$, respectivamente (tab. I). As fêmeas foram menos receptíveis à alimentação oferecida, o que segundo GibBons \& Semlitsch (1987) pode estar relacionado com a fase do ciclo reprodutivo, já que a maioria das fêmeas de M. corallinus fez postura de ovos logo após a entrada no biotério e em alguns destes ovos o desenvolvimento era incompleto, o que sugere a influência do fator estresse provocado pelo cativeiro.

Os resultados obtidos com M. corallinus, que receberam alimentação forçada mensalmente, mostraram-se insatisfatórios, pois os animais foram mais susceptíveis à ação de agentes patogênicos como Staphylococcus aureus (Rosenbach, 1884) e Pseudomonas aeruginosa (Schroeter, 1885) (50,0\%), quando comparados às serpentes que se alimentaram voluntariamente $(18,7 \%)$. O número de óbitos nas serpentes que desenvolveram algum tipo de patologia, como dermatite necrótica e infecção sistêmica, 


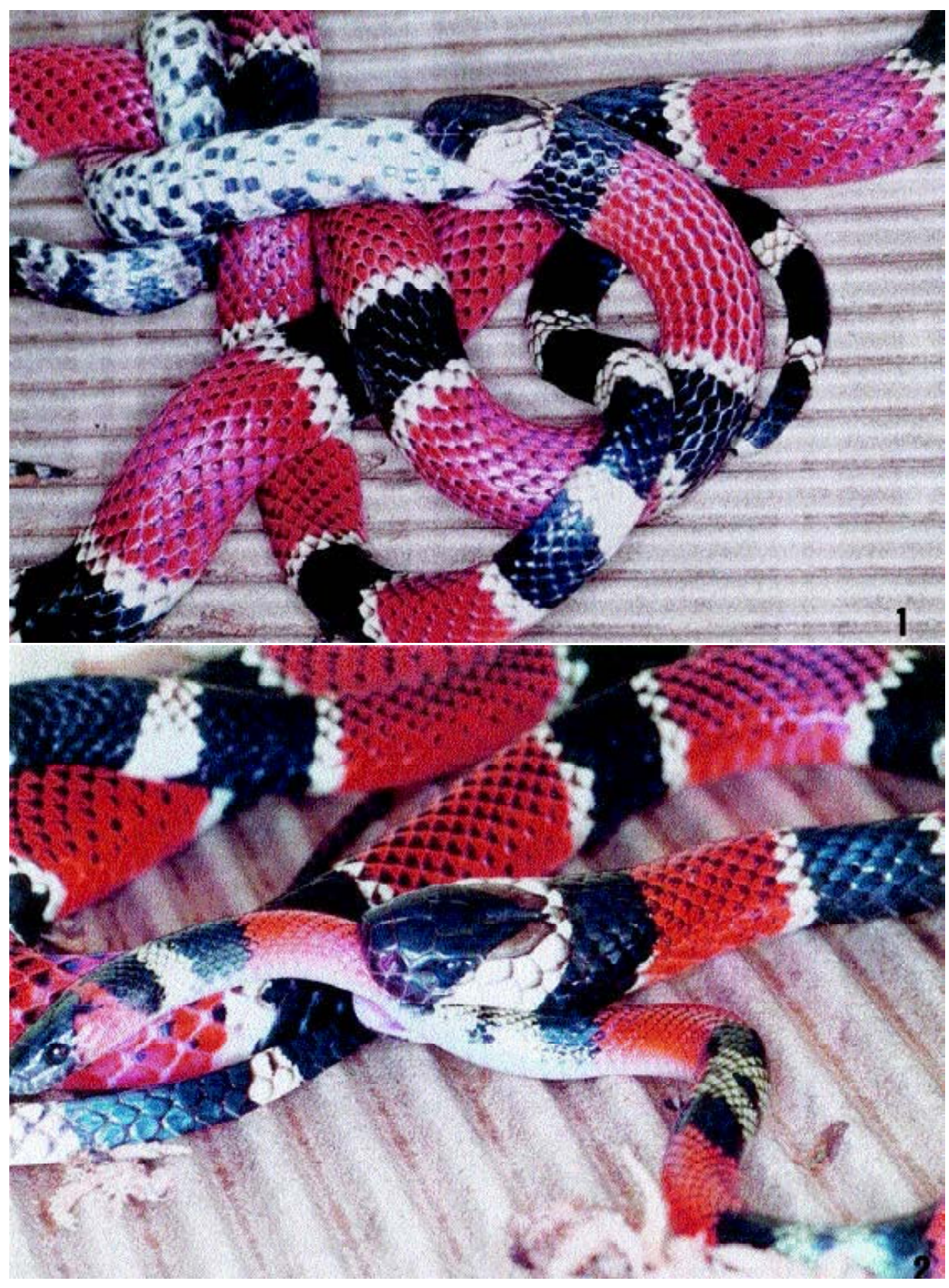

Figs. 1, 2. Micrurus corallinus (Merrem,1820) (comprimento rostro-cloacal 730-850 mm): 1, ingerindo Sibynomorphus mikanii (Schlegel, 1837); 2, ingerindo Oxyrhopus guibei (Duméril \& Bibron Duméril, 1854). Fotos: Alexandre Pinheiro Zanotti. 
pneumonia e parasitoses, também foi maior no grupo B, representando $37,5 \%$, enquanto no grupo A, apenas $12,5 \%$ das serpentes foram a óbito nas mesmas circunstâncias. Segundo Mishima et al. (1972), a alimentação forçada é responsável pelo aumento da susceptibilidade à ação dos agentes patogênicos, devido à contaminação pela introdução da sonda, por via esofágica.

Segundo CAMPBell (1973) M. fulvius tenere (Baird \& Girard, 1853) apresentou boa aceitação para alimentos congelados, como pequenos lagartos e serpentes. SoftLY \& СоскEтT (1966) relataram que a utilização de alimentos previamente congelados pode reduzir a transmissão de parasitas, para as serpentes mantidas em cativeiro. Em condições cativas, MARQuES \& SAZIMA (1997) observaram que M. corallinus ingeriu dois espécimes recém-mortos de Leposternon microcephalum (Wagler, 1824).

$\mathrm{O}$ manuseio e a anestesia das serpentes são procedimentos necessários para a alimentação forçada, mas também podem causar desconforto e estresse às serpentes, resultando em regurgitação do alimento (AsHLEY \& BURCHFIELD, 1968).

A freqüência da alimentação de serpentes é dependente da espécie e das condições ambientais, ou seja, espécies mais ativas necessitam de alimento com maior freqüência que as espécies lentas. Espécies que são mantidas em ambientes aquecidos digerem mais rapidamente o alimento do que aquelas mantidas em níveis baixos de temperatura (CunNingham \& GiLI, 1992). Em M. corallinus a indução mensal de alimento mostrou-se ineficaz. Alimentações quinzenais ou semanais seriam indesejáveis, pois são freqüentes os casos de óbitos após o manuseio dos animais, a anestesia com $\mathrm{CO}_{2}$, extração de veneno, alimentação forçada, contenção para exames clínicos ou aplicação de medicamentos, entre outros.

Mishima et al. (1972) realizaram um estudo experimental com várias espécies de

Tabela I. Relação entre a oferta e ingestão voluntária (grupo A) de exemplares de colubrídeos mais aceitos pelas serpentes Micrurus corallinus (número de colubrídeos oferecidos/número de colubrídeos ingeridos; $\mathrm{M}$, macho; $\mathrm{F}$, fêmea).

\begin{tabular}{cccc}
\hline Amostra/Sex & $\begin{array}{c}\text { Sibynomorphus spp. } \\
\text { oferta/ingestão }\end{array}$ & $\begin{array}{c}\text { Oxyrhopus guibei } \\
\text { oferta/ingestão }\end{array}$ & $\begin{array}{c}\text { Liophis spp. } \\
\text { oferta/ingestão }\end{array}$ \\
\hline $1 / \mathrm{F}$ & $1 / 1$ & $1 / 0$ & $3 / 3$ \\
$2 / \mathrm{M}$ & $7 / 1$ & $8 / 6$ & $2 / 1$ \\
$3 / \mathrm{F}$ & - & $1 / 0$ & $2 / 0$ \\
$4 / \mathrm{M}$ & $2 / 1$ & $5 / 2$ & $1 / 1$ \\
$5 / \mathrm{M}$ & $2 / 2$ & $5 / 3$ & - \\
$6 / \mathrm{M}$ & $9 / 5$ & $7 / 4$ & - \\
$7 / \mathrm{M}$ & $2 / 1$ & $1 / 0$ & $3 / 1$ \\
$8 / \mathrm{M}$ & $5 / 5$ & $1 / 1$ & $1 / 1$ \\
$9 / \mathrm{F}$ & $9 / 7$ & $4 / 3$ & $1 / 1$ \\
$10 / \mathrm{F}$ & - & - & $2 / 0$ \\
$11 / \mathrm{M}$ & $20 / 16$ & $16 / 9$ & $3 / 1$ \\
$12 / \mathrm{M}$ & $8 / 7$ & $5 / 5$ & $2 / 2$ \\
$13 / \mathrm{M}$ & $5 / 4$ & - & $3 / 2$ \\
$14 / \mathrm{F}$ & $2 / 0$ & $1 / 0$ & $1 / 0$ \\
$15 / \mathrm{F}$ & - & - & - \\
$16 / \mathrm{F}$ & $4 / 0$ & - & - \\
\hline Total & $76 / 50$ & $55 / 3$ & $24 / 13$ \\
\hline$\%$ & 65,8 & 60,0 & 54,2 \\
\hline
\end{tabular}


serpentes de regiões temperadas e concluíram que a alimentação voluntária foi mais favorável para as espécies cativas que a forçada. Os autores sugerem que a indução de alimento deva ser utilizada como um recurso para aquelas serpentes que não aceitam a alimentação natural em ambiente cativo. Em nosso estudo, a alimentação voluntária também foi considerada a mais indicada para $M$. corallinus, visto que os animais mostraram-se mais resistentes à ação dos agentes patogênicos.

Agradecimentos. Aos pesquisadores do Instituto Butantan, Maria de Fátima D. Furtado, Hana Suzuki e Thélia R. F. J. Cinquini pelas críticas e sugestões, a Wilson Fernandes pelo auxílio no experimento, a Otávio A. V. Marques, pelo aporte de informação bibliográfica e a Alexandre P. Zanotti, pela elaboração fotográfica.

\section{REFERÊNCIAS BIBLIOGRÁFICAS}

AmARAL, A. 1978. Serpentes do Brasil: Iconografia colorida. Brasília, Melhoramentos. 220p. Ashley, B. D. \& Burchfield, P. M. 1968. Maintenance of a snake colony for the purpose of venom extraction. Toxicon, London, 5:267-275.

Campbell, J. A. 1973. A captive hatching of Micrurus fulvius tenere (Snake, Elapidae). J. Herpetol., Kansas, 7(3):312-315.

Cunningham, A. A. \& Gili, C. 1992. Management en captivity. In: Beynon, P. H.; Lawton, M. P. C. \& COOPER, J. E. Manual of reptiles. Iowa, British Small Animal Veterinary Association. p.14-31.

Gans, C. 1969. Amphisbaenians, reptiles specialized for a burrowing existence. Endeavour, London, 28: $146-96$

. 1974. Biomechanics: an approach to vertebrate biology. Toronto, J. B. Lippincott. p.117191.

Gibbons, J. W. \& Semlitsch, R. D. 1987. Activity pattern. In: Seigel, R. A.; Collins, J. T. \& NovaK, S. S. eds. Snakes: ecology and evolutionary biology. New York, McGraw-Hill. p.396-421.

Marques, O. A. V. \& Sazima, I. 1997. Diet and feeding behaviour of the coral snake, Micrurus corallinus, from the Atlantic Forest of Brazil. Herpetol. Nat. Hist., Stanford, 5(1):88-93.

Mishima, S.; Lin, Y. F. et al. 1972. Experimental studies on the extraction and force-feeding of some venomous snakes. The Snake, Gunma, 4:51-58.

Roze, J. 1996. Coral snakes of the Americas. Biology, identification and venoms. Florida Krieger Publ. 328p.

Softly, A. \& Cockett, E. G. 1966. Aspects of maintaining snakes as laboratory animals. J. Inst. Anim. Techn., Nottingham, 17(2):47-60.

Recebido em 15.04.2002; aceito em 11.10.2002 